\title{
Different response rates to chemotherapy between Japanese and German esophageal squamous cell carcinoma: patients may be influenced by $E R C C 1$ or $A B C B 1$
}

\author{
Kosuke Narumiya*,1 (D), Elfriede Bollschweiler2, Arnulf H Hölscher²,3, Masakazu \\ Yamamoto $^{1}$, Uta Drebber ${ }^{4}$, Hakan Alakus ${ }^{5}$, Ralf Metzger ${ }^{6}$ \& Ute Warnecke-Eberz ${ }^{5}$ \\ ${ }^{1}$ Department of Surgery, Institute of Gastroenterology, Tokyo Women's Medical University, Kawada 8-1, \\ Shinjyuku, Tokyo, 1628666, Japan \\ ${ }^{2}$ Medical Faculty, University of Cologne, Kerpener Str. 62, Cologne, 50924, Germany \\ ${ }^{3}$ Head Contilia Center for Esophageal Diseases, Elisabeth Hospital, Klara-Kopp-Weg 1, Essen, 45138, Germany \\ ${ }^{4}$ Institute of Pathology, University of Cologne, Kerpener Str. 62, Cologne, 50924, Germany \\ ${ }^{5}$ Department of General, Visceral \& Cancer Surgery, University of Cologne, Kerpner Str. 62, Cologne, 50924, Germany \\ ${ }^{6}$ Department of General, Visceral, Thoracic \& Cancer Surgery, Caritas Klinikum, Rhine Str. 2, Saarbrücken, 66113, Germany \\ *Author for correspondence: Tel.: +81 33353 8111; Fax: +81 35269 7507; narumiya.ige@twmu.ac.jp
}

\begin{abstract}
Aim: To find out differences in biomarkers between Japanese and German patients responsible for response after neoadjuvant radio/chemotherapy and survival for esophageal squamous cell carcinoma. Materials \& methods: A total of 60 patients from Japan and 127 patients from Germany with esophageal squamous cell carcinoma were analyzed according to three SNPs by real-time PCR. Results: The distribution of the genotypes of ERCC1 rs16115 and $A B C B 1$ C3435T rs 1045642 was significantly different between both patients' groups. Japanese patients had significantly less good response to 5 -fluorouracil/cisplatin chemotherapy. The influence of the three SNPs on response varied between patients from Japan and Germany. Conclusion: Different expressions of ERCC1 and $A B C B 1$ SNPs of Japanese patients compared with the German patients partially explain the different response.
\end{abstract}

First draft submitted: 19 May 2020; Accepted for publication: 16 June 2020; Published online:

2 July 2020

Keywords: $A B C B 1$ • biomarker • ERCC1 • German • GNAS • Japanese • molecular biology • neoadjuvant chemotherapy • single-nucleotide polymorphisms • squamous cell carcinoma of the esophagus

The incidence of squamous cell carcinoma of the esophagus (ESCC) differs between Japan and Germany. In 2005 the age-standardized incidence rate per year of ESCC was 9.2 in Japan and 3.9 in Germany per 100,000 males [1,2]. In addition, there is abundant evidence that nationality is associated with differences in survival time and drug sensitivity [3-5]. The standard treatment for patients with resectable ESCC is transthoracic esophagectomy with two- or three-field lymphadenectomy [6]. Patients with advanced esophageal cancer receive increasingly more preoperative chemotherapy or chemoradiation $[7,8]$. But, the therapeutic guidelines for patients with ESCC vary between Germany and Japan [9,10].

Different factors are known to influence drug response, such as age, gender, ethnicity, comorbidity and especially genetics [11]. A great number of molecular markers could be identified as potentially predictive and prognostic markers for patients with neoadjuvant therapy for different solid tumors, including esophageal cancer $[12,13]$. One kind of such biological markers are SNPs. There are several reports suggesting the involvement of SNPs in drug metabolism and to the variability of drug response $[13,14]$.

In earlier studies we analyzed three different polymorphisms which had significant influence on prognosis and/or response to preoperative chemoradiation.

Of these, the multidrug resistance gene, $A B C B 1$, seems to play an important role in pharmacogenetics. $A B C B 1$ is a member of the ATP-binding cassette superfamily of membrane transporters. The overexpression of this gene

Future Medicine 
may be one reason for the resistance to chemotherapeutic agents like 5-fluorouracil (5-FU) and cisplatin in cancer patients [13,15]. The data of an earlier study support the role of $A B C B 1$ rs 1045642 SNP in treatment response of esophageal cancer. SNPs of $A B C B 1$ could be helpful to predict lymph node regression in multimodality treatment of locally advanced esophageal cancer [16].

Our group's research focus has been the characterization of predictive factors for neoadjuvant therapy of esophageal cancer [17-20]. One of our most recent projects investigated gene polymorphism of ERCC1 to predict response to neoadjuvant chemoradiation (cisplatin, 5-FU, $40 \mathrm{~Gy}$ ) in advanced esophageal cancer [21]. The results of the prospective study confirmed that ERCC1 gene polymorphisms are predictive for histopathological minor response to cisplatin/5-FU-based chemoradiation in esophageal carcinoma [22].

The results of another study showed that a group of patients with advanced esophageal cancer - those with a homozygous GNAS 393C genotype - can be excluded from neoadjuvant therapy because of low response rate and no prognostic benefit [23].

The aim of the study was to find out reasons for the published differences in chemotherapeutic drug sensitivity and survival rate between Japanese and Caucasian patients with esophageal cancer. We hypothesized that the explanation are the differences in molecular cancer biology modifying the rate of response to chemotherapy. Therefore, we analyzed the possible influence of the three SNPs (ERCC1 rs1615, ABCB1 C3435T rs1045642 and $G$-protein rs7121) to the therapeutic results of ESCC patients with comparable therapeutic procedures (transthoracic esophagectomy after neoadjuvant therapy) using the data of patients from Japan and Germany.

\section{Patients \& methods}

Study patients from Japan

A total of 60 patients from Japan with advanced ESCC were available for this retrospective study. Esophagectomy was performed between 1998 and 2003 in the Department of Surgery, Institute of Gastroenterology Tokyo Women's Medical University (Tokyo, Japan). All patients underwent conventional thoracotomy and laparotomy including two-field lymphadenectomy of mediastinal and abdominal lymph nodes. Extended esophagectomy was performed through a right thoracotomy, with patients in the left lateral position. After esophagectomy, abdominal lymph node dissection and reconstruction using a gastric tube were done with patients in the supine position. The anastomosis was performed in the neck via a left cervical incision. Three-field lymph node dissection was indicated for upper thoracic cancer, cervical lymph node metastasis and superior mediastinal node metastasis on pre- or intraoperative findings especially detected by ultrasonography. Clinical data are shown in Table 1.

None of the included patients received prior radio- and/or chemotherapy for other diseases. A total of 20 patients received primary surgery without neoadjuvant treatment (Table 1). The indication for neoadjuvant chemotherapy was extended spread of regional lymph node metastasis $(\mathrm{cTxN}+\mathrm{M} 0)$. Neoadjuvant chemoradiotherapy was established in patients with locally advanced tumor (cT3NxM0). In the case of chemotherapy, cisplatin $\left(80 \mathrm{mg} / \mathrm{m}^{2} /\right.$ day) was administered as a continuous infusion over $24 \mathrm{~h}$ on day 1 and day 29 and 5 -FU $\left(800 \mathrm{mg} / \mathrm{m}^{2} /\right.$ day $)$ as a continuous infusion over $24 \mathrm{~h}$ on days $1-5$ and $29-33$. In the case of chemoradiotherapy, cisplatin $\left(70 \mathrm{mg} / \mathrm{m}^{2} /\right.$ day $)$ was administered as a continuous infusion over $24 \mathrm{~h}$ on day 1 and $5-\mathrm{FU}\left(700 \mathrm{mg} / \mathrm{m}^{2} /\right.$ day $)$ as a continuous infusion over $24 \mathrm{~h}$ on days $1-4$. Radiation therapy was administered by linear accelerators. Radiation therapy was planned to encompass the tumor volume with $2 \mathrm{~cm}$ radial margins and the planning target volume included enlarged regional nodes based on computed tomography (CT) evaluation. Radiation was delivered in daily fractions of 2.0 Gy (days $1-5,8-12,15-19$ and 22-26) to a total dose of 40 Gy using a multiple field technique. Surgical resection was performed 4-6 weeks following completion of chemoradiation, after clinical restaging.

\section{Study patients from Germany}

We analyzed formalin-fixed and paraffin-embedded material of 127 patients with clinical T3NxM0 ESCC that underwent resection after neoadjuvant therapy between 1997 and 2008 at the Department of General, Visceral and Cancer Surgery, University of Cologne (Cologne, Germany). Surgery for patients with ESCC was laparotomic or laparoscopic gastrolysis followed by right transthoracic en bloc esophagectomy including two-field lymphadenectomy of mediastinal and abdominal lymph nodes. This surgical procedure and the reconstruction with high intrathoracic esophagogastrostomy was described more detailed previously [7]. Clinical data are shown in Table 1.

None of the patients had undergone previous radiotherapy and/or chemotherapy for other diseases. All patients received preoperative chemoradiation. Briefly, cisplatin $\left(20 \mathrm{mg} / \mathrm{m}^{2} /\right.$ day $)$ was administered as a short-term infusion on days 1 to 5 and $5-\mathrm{FU}\left(1000 \mathrm{mg} / \mathrm{m}^{2} /\right.$ day $)$ was given as continuous infusion over $24 \mathrm{~h}$ on days $1-5$. Radiation 


\begin{tabular}{|c|c|c|c|c|c|}
\hline 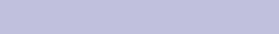 & Japanese & & German & & Significance Japanese-German \\
\hline Total & $\mathrm{n}=60$ & & $\mathrm{n}=127$ & & - \\
\hline Age (median) & 61 years & & 60 years & & NS \\
\hline Min-max & $46-79$ years & & $29-80$ years & & \\
\hline Gender & & & & & $p=0.008$ \\
\hline Male & 52 & $87 \%$ & 87 & $68 \%$ & \\
\hline Female & 8 & $13 \%$ & 40 & $32 \%$ & \\
\hline T-category & & & All cT3-4 & & - \\
\hline pT1-2 & $\mathrm{n}=10$ & & & & \\
\hline pT3 & $\mathrm{n}=10$ & & & & \\
\hline урт0-1 & $\mathrm{n}=7$ & & 46 & $36 \%$ & \\
\hline урт2 & $n=5$ & & 28 & $22 \%$ & \\
\hline урт3-4 & $\mathrm{n}=28$ & & 53 & $42 \%$ & \\
\hline Therapy & & & & & - \\
\hline Surgery & $\mathrm{n}=20$ & & 0 & & \\
\hline $\mathrm{CT} x+\mathrm{S}$ & $\mathrm{n}=20$ & & 0 & & \\
\hline $\mathrm{RT} x / \mathrm{CT} x+\mathrm{S}$ & $n=20$ & & $n=127$ & & \\
\hline ERCC1 rs11615 & & & & & $\mathrm{p}<0.0001$ \\
\hline CC & 28 & $47 \%$ & 17 & $13 \%$ & \\
\hline $\mathrm{TT}$ & 7 & $12 \%$ & 52 & $41 \%$ & \\
\hline $\mathrm{C} / \mathrm{T}$ & 25 & $41 \%$ & 58 & $46 \%$ & \\
\hline ABCB1 C3435T rs 1045642 & & & & & $p=0.0002$ \\
\hline $\mathrm{CC}$ & 31 & $52 \%$ & 28 & $22 \%$ & \\
\hline $\mathrm{TT}$ & 8 & $13 \%$ & 32 & $25 \%$ & \\
\hline $\mathrm{C} / \mathrm{T}$ & 21 & $35 \%$ & 67 & $53 \%$ & \\
\hline GNAS T393C rs7121 & & & & & NS \\
\hline CC & 12 & $20 \%$ & 39 & $31 \%$ & \\
\hline $\mathrm{TT}$ & 14 & $23 \%$ & 24 & $19 \%$ & \\
\hline $\mathrm{C} / \mathrm{T}$ & 34 & $57 \%$ & 64 & $50 \%$ & \\
\hline
\end{tabular}

was delivered in daily fractions of 1.8 Gy to a total dose of 40 Gy using a multiple field technique. Clinical restaging by endoscopy and CT after induction therapy was performed in all patients to exclude distant metastasis.

\section{Histopathologic response classification}

All resected specimens were examined histopathologically in the Department of Pathology, University of Cologne, determining tumor stage, residual tumor category $(\mathrm{R})$, histopathologic grading $(\mathrm{G})$ and number of examined and involved lymph nodes (LN). The results were classified according to the seventh edition of the TNM (tumor-nodemetastasis) classification. The histopathologic workup of the entire primary tumor was performed as previously described [7]. The histopathologic regression of the primary tumor was evaluated by quantifying therapy-induced changes including reactive changes such as necrosis, fibrosis, foamy histiocytes and giant cell reactions. The degree of histomorphologic regression was classified into four categories (Cologne Regression Scale): grade IV, complete response; grade III, $<10 \%$ vital residual tumor cells (VRTCs); grade II, $10 \%$ to $50 \%$ VRTCs; and grade I, $>50 \%$ VRTCs [24]. Figure 1 showed examples for histopathologic evaluation of tumor regression in esophageal squamous cell carcinoma.

In addition, the primary tumor was classified as ypT-category according to the Union for International Cancer Control (UICC)-TNM classification. Response to neoadjuvant therapy was also analyzed for the resected lymph nodes and quantified as the frequency of patients without lymph node metastasis after neoadjuvant therapy (ypN0). This analysis was performed by experienced staff pathologists blinded to all other clinical data.

\section{Genotyping}

DNA was extracted from paraffin-embedded tissues from resection boundaries containing exclusively normal cells using the QIAamp kit (Qiagen, Hilden, Germany). Genomic DNA was used for detection of SNPs by real-time CR by TaqMan 7900HT (Applied Biosystems, Darmstadt, Germany).

Genotyping for ERCC1 rs11615 SNP, $A B C B 1$ rs1045642 SNP and GNAS T393C rs7121 SNP was subdifferentiated into homozygous $\mathrm{C}$-allele $(\mathrm{CC})$ and T-allele (TT) and heterozygous $\mathrm{C} / \mathrm{T}$ carriers. 


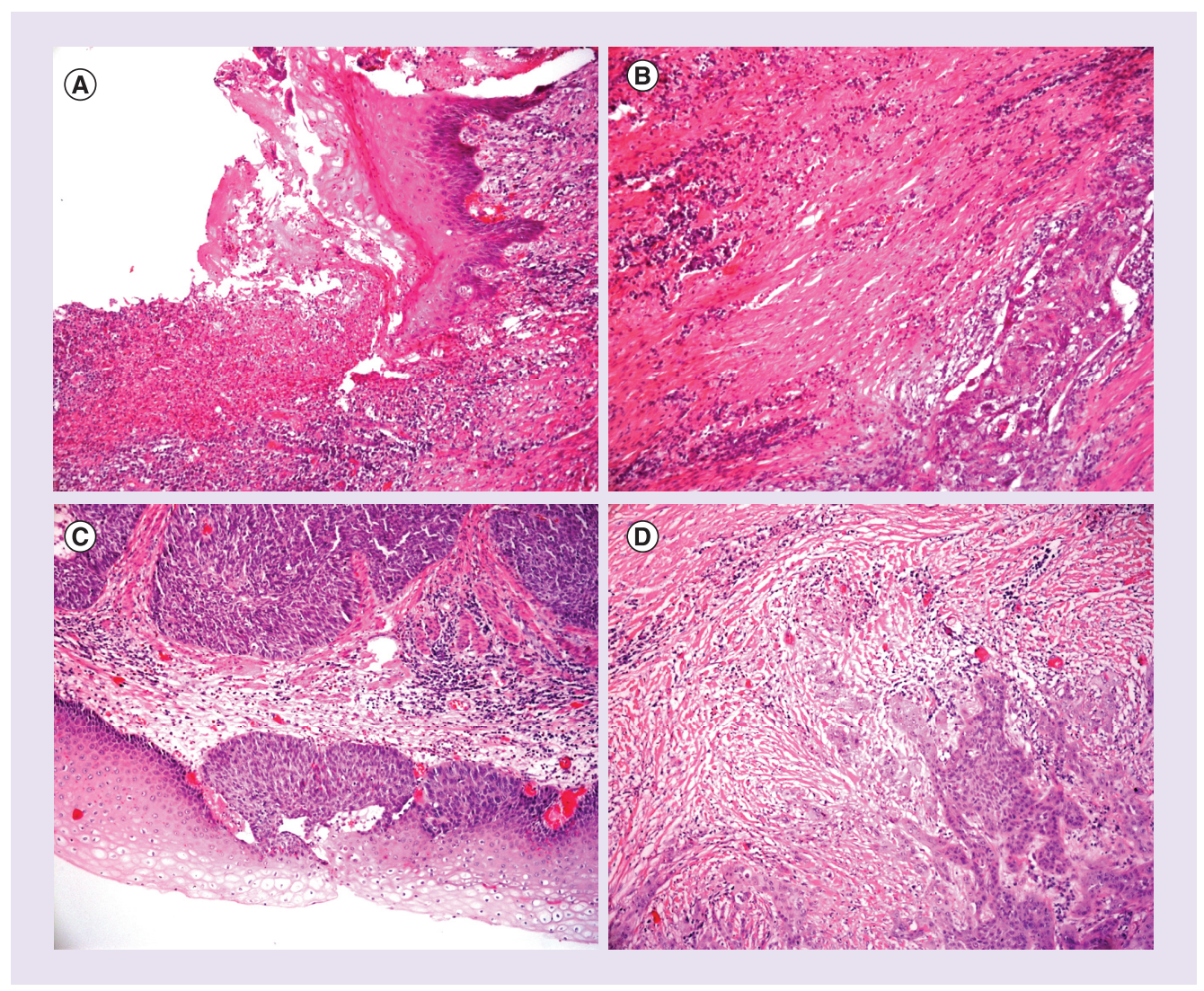

Figure 1. Examples of the histomorphologic evaluation of tumor regression after neoadjuvant chemoradiation in patients with advanced esophageal squamous cell carcinoma using the Cologne Regression Scale. Case $1=$ ESCC with grade III defined as less than $10 \%$ VTCs; (A) ulcera with powerful signs of regression and no VTCs. (B) About $8 \%$ VTCs in the depths. Case 2 = ESCC with grade I defined as more than $50 \%$ VTCs; (C) tumor with only small signs of regression, about $90 \%$ VTCs. (D) Border of the tumor - again, only low regression, about $95 \%$ VTCs. ESCC: Esophageal squamous cell carcinoma; VTC: Vital tumor cell.

\section{Statistics}

The median, with its range, was used for descriptive statistics. Factor frequencies were calculated using chi-square statistics with a significance level of $\mathrm{p}<0.05$.

Kaplan-Meier survival calculations were used to depict survival distribution and the median survival rates with 95\% CI were computed. The log-rank test was applied to evaluate survival differences. We analyzed the overall survival without differentiation between tumor-related death or death from other causes.

The analyses of prognosis were carried out using the statistics program MedCalc Statistical Software version 18.2.1 (MedCalc Software bvba, Ostend, Belgium; https://www.medcalc.org; 2018) was used.

This retrospective study was performed according to the criteria of the ethics committee of the University Hospital of Cologne or of the ethics committee of Tokyo Women's Medical University.

\section{Results}

We compared the data from 60 patients with ESCC from Japan with those from 127 patients with ESCC from Germany. The clinical data are shown in Table 1.

\section{ERCC1, $A B C B 1$ \& GNAS SNP in ESCC patients}

Patients from Japan had significantly more often homozygous CC ERCC1 rs11615 and less TT genotype compared with German patients ( $\mathrm{p}<0.0001$ ). The distribution of the genotypes of $A B C B 1$ C3435T rs1045642 was significantly different between German and Japanese patients $(\mathrm{p}=0.0002)$. The homozygous CC and TT as well as the heterozygous C/T genotypes of GNAS rs7121 in patients from Japan and Germany were not different (Table 1). 


\begin{tabular}{|c|c|c|c|c|c|c|}
\hline SNP & $\begin{array}{l}\text { Japanese normal } \\
\text { population }\end{array}$ & $\begin{array}{l}\text { Japanese patients } \\
\text { with ESCC }\end{array}$ & Significance & $\begin{array}{l}\text { European normal } \\
\text { population }\end{array}$ & $\begin{array}{l}\text { German patients with } \\
\text { ESCC }\end{array}$ & Significance \\
\hline rs11615 & $\mathrm{n}=122^{\dagger}$ & $\mathrm{n}=60$ & & $\mathrm{n}=647^{\ddagger}$ & $\mathrm{n}=127$ & \\
\hline ERCC1 & & & NS & & & NS \\
\hline CC & $50 \%$ & $47 \%$ & & $13 \%$ & $13 \%$ & \\
\hline TT & $8 \%$ & $12 \%$ & & $42 \%$ & $41 \%$ & \\
\hline$A B C B 1$ C3435T & & & $p=0.014$ & & & NS \\
\hline $\mathrm{CC}$ & $31 \%$ & $52 \%$ & & $15 \%$ & $22 \%$ & \\
\hline TT & $16 \%$ & $13 \%$ & & $29 \%$ & $25 \%$ & \\
\hline $\mathrm{C} / \mathrm{T}$ & $52 \%$ & $35 \%$ & & $56 \%$ & $53 \%$ & \\
\hline rs7121 & $\mathrm{n}=90$ & $\mathrm{n}=60$ & & $\mathrm{n}=800^{\mathbb{I}}$ & $n=127$ & \\
\hline
\end{tabular}

\section{ERCC1, $A B C B 1$ \& GNAS SNP in normal populations}

We used published data for the three SNPs from Japanese and European normal populations $[23,25,26]$. Table 2 shows the frequency of the SNPs in both normal populations. Comparing the distribution of genotypes in the Japanese and European normal populations, Japanese individuals had significantly more often homozygous CC ERCC1 rs11615 (50 vs $13 \%$ ) and less TT genotype (8 vs $42 \%$ ) than German individuals ( $<<0.0001)$. Similar results were seen for $A B C B 1$ C 3435 T rs 1045642 with a frequency of 31 versus $15 \%$ for CC genotype, 16 versus $29 \%$ for TT genotype and 52 versus $56 \%$ for $\mathrm{C} / \mathrm{T}$ genotype comparing the Japanese and European normal populations $(\mathrm{p}<0.0001)$. The distribution of the genotypes of GNAS SNP was not different between the Japanese and German normal populations.

\section{ERCC1, $A B C B 1$ \& GNAS SNP in normal populations \& ESCC patients}

The distribution of the genotypes of ERCC1 rs11615 SNP as well as of GNAS T393C rs7121 was not different between the normal populations and ESCC patients in Japan and in Germany. Only the frequency of $A B C B 1$ $\mathrm{CC}$ and CT genotype was significantly different between the Japanese normal population and ESCC patients $(\mathrm{p}=0.014)$. Homozygous genotype CC of $A B C B 1$ may be a risk factor for ESCC.

\section{Response to neoadjuvant chemotherapy in Japanese \& German ESCC patients}

We analyzed the data of patients with advanced squamous cell carcinoma treated with neoadjuvant therapy and compared 40 patients from Japan (chemotherapy or chemoradiation) with 127 patients from Germany (chemoradiation). Response to neoadjuvant therapy was analyzed from the primary tumor and via the frequency of patients without lymph node metastasis after neoadjuvant therapy (ypN0).

Comparing the rate of patients with complete or nearly complete response (ypT0-1) we found significant differences between Japanese and German patients (Table 3). The frequency of ypT-category was ypT0-1 = 18\%, $\mathrm{ypT} 2=13 \%$ and $\mathrm{ypT} 3-4=69 \%$ in Japanese patients and ypT0-1 $=36 \%$, ypT2 $=22 \%$ and $\mathrm{ypT} 3-4=42 \%$ in German patients with p-trend $=0.02$ for more advanced ypT-categories in Japanese patients. The rate of ypT0-1 was not significantly different after chemotherapy or chemoradiation in Japanese patients $(\mathrm{p}=0.119)$.

In addition, we analyzed the rate of non-response to neoadjuvant therapy as less than $50 \%$ reduction of the primary tumor or progression (grade I of Cologne Regression Scale). Only 20 of 127 (16\%) patients from Germany showed non-response to neoadjuvant therapy compared with Japanese patients $(21$ of $40,52 \%)(\mathrm{p}<0.0001)$. The rate of grade I was not significantly different after chemotherapy or chemoradiation in Japanese patients $(\mathrm{p}=0.118)$. 
Table 3. Rate of response to neoadjuvant therapy comparing Japanese and German cT3 esophageal squamous cell carcinoma patients (vital tumor cells, grade I-IV histopathologic tumor regression according to Cologne Regression Scale).

\begin{tabular}{|c|c|c|c|c|c|}
\hline & \multicolumn{2}{|c|}{ Japanese } & \multicolumn{2}{|c|}{ German } & \multirow{2}{*}{$\begin{array}{l}\text { Significance } \\
\text { p-value }\end{array}$} \\
\hline & n & $\%$ & n & $\%$ & \\
\hline Total & 40 & $100 \%$ & 127 & $100 \%$ & - \\
\hline ypT-category & & & & & $p=0.027$ \\
\hline урт0-1 & 7 & $18 \%$ & 46 & $36 \%$ & \\
\hline урт2-4 & 33 & $82 \%$ & 81 & $64 \%$ & \\
\hline Histopathologic response & & & & & $p<0.0001$ \\
\hline$<=50 \%$ VTCs grade II-IV & 19 & $48 \%$ & 107 & $84 \%$ & \\
\hline$>50 \%$ VTCs or progression grade I & 21 & $52 \%$ & 20 & $26 \%$ & \\
\hline ypN-category & & & & & $p=0.006$ \\
\hline ypNO & 11 & $28 \%$ & 46 & $54 \%$ & \\
\hline ypN1-3 & 29 & $72 \%$ & 81 & $46 \%$ & \\
\hline
\end{tabular}

\begin{tabular}{|c|c|c|c|c|c|c|c|c|c|c|}
\hline & \multirow{3}{*}{ Japanese } & \multicolumn{4}{|c|}{ Response } & \multirow{3}{*}{ German } & \multicolumn{4}{|c|}{ Response } \\
\hline & & \multicolumn{2}{|c|}{ урт0-1 } & \multicolumn{2}{|c|}{ ypNO } & & \multicolumn{2}{|c|}{ урт0-1 } & \multicolumn{2}{|c|}{ ypNO } \\
\hline & & $n$ & $\%$ & $n$ & $\%$ & & $n$ & $\%$ & $n$ & $\%$ \\
\hline Total & $\mathrm{n}=40$ & 7 & $18 \%$ & 11 & $28 \%$ & $\mathrm{n}=127$ & 46 & $36 \%$ & 68 & $54 \%$ \\
\hline \multicolumn{11}{|c|}{ ERCC1 rs11615 } \\
\hline $\mathrm{CC}$ & 17 & 2 & $12 \%$ & 5 & $29 \%$ & 17 & 6 & $35 \%$ & 6 & $29 \%$ \\
\hline TT & 6 & 2 & $33 \%$ & 2 & $33 \%$ & 52 & 14 & $25 \%$ & 24 & $48 \%$ \\
\hline $\mathrm{C} / \mathrm{T}$ & 17 & 3 & $18 \%$ & 4 & $23 \%$ & 58 & 26 & $45 \%$ & 39 & $67 \%$ \\
\hline \multicolumn{11}{|c|}{ ABCB1 C3435T rs 1045642} \\
\hline $\mathrm{CC}$ & 25 & 5 & $20 \%$ & 5 & $20 \%$ & 28 & 12 & $42 \%$ & 12 & $43 \%$ \\
\hline $\mathrm{TT}$ & 3 & 1 & $33 \%$ & 3 & $100 \%$ & 32 & 11 & $34 \%$ & 21 & $66 \%$ \\
\hline $\mathrm{C} / \mathrm{T}$ & 12 & 1 & $8 \%$ & 3 & $25 \%$ & 67 & 23 & $34 \%$ & 36 & $54 \%$ \\
\hline \multicolumn{11}{|c|}{ GNAS - T393C rs7121 } \\
\hline $\mathrm{CC}$ & 9 & 2 & $22 \%$ & 5 & $56 \%$ & 39 & 10 & $26 \%$ & 19 & $49 \%$ \\
\hline TT & 11 & 2 & $18 \%$ & 2 & $18 \%$ & 24 & 9 & $38 \%$ & 12 & $50 \%$ \\
\hline $\mathrm{C} / \mathrm{T}$ & 20 & 3 & $15 \%$ & 4 & $20 \%$ & 64 & 26 & $41 \%$ & 37 & $58 \%$ \\
\hline
\end{tabular}

The frequency of patients without lymph node metastases after neoadjuvant therapy (ypN0) is a further measure for response to preoperative therapy. A total of $54 \%$ of German patients and $28 \%$ of Japanese patients had ypN0-category $(\mathrm{p}=0.006)$.

\section{Correlation of SNPs \& response}

Comparing the rate of ypT0-1 for the different genotypes of ERCC1, ABCB1 and GNAS we found no significant differences between Japanese and German patients (Table 4).

The subgroup analysis of the genotypes of the three SNP showed higher rate of nonresponse in Japanese patients for:

- ERCC1 rs11615 CC with 59\% in Japanese versus $18 \%$ in German patients (p = 0.032);

- ERCC1 rs11615 C/T with 65\% in Japanese versus 30\% in German patients (p = 0.011);

- $A B C B 1$ C3435T rs1045642 C/T with 67\% in Japanese versus $18 \%$ in German patients $(\mathrm{p}<0.002)$;

- GNAS T393C rs7121 CC with 56\% in Japanese versus 16\% in German patients (p = 0.020).

Comparing the frequency of the various genotypes in the group of non-responders with those of all patients only the data for ABCB1 in the group of German patients showed significant differences (Table 5).

The multivariate regression analysis showed that the influence of these SNPs on response varies between Japanese and German patients (Figure 2). For example, Japanese patients with C/T ERCC1 and C/T ABCB1 had the 
Table 5. Frequency of genotypes of three SNP comparing all patients and those with histopathologic nonresponse to neoadjuvant therapy (less than $50 \%$ reduction of the primary tumor after neoadjuvant therapy = grade I according to Cologne Regression Scale) in Japanese and German (T3 esophageal squamous cell carcinoma patients comparing.

NS: Not significant

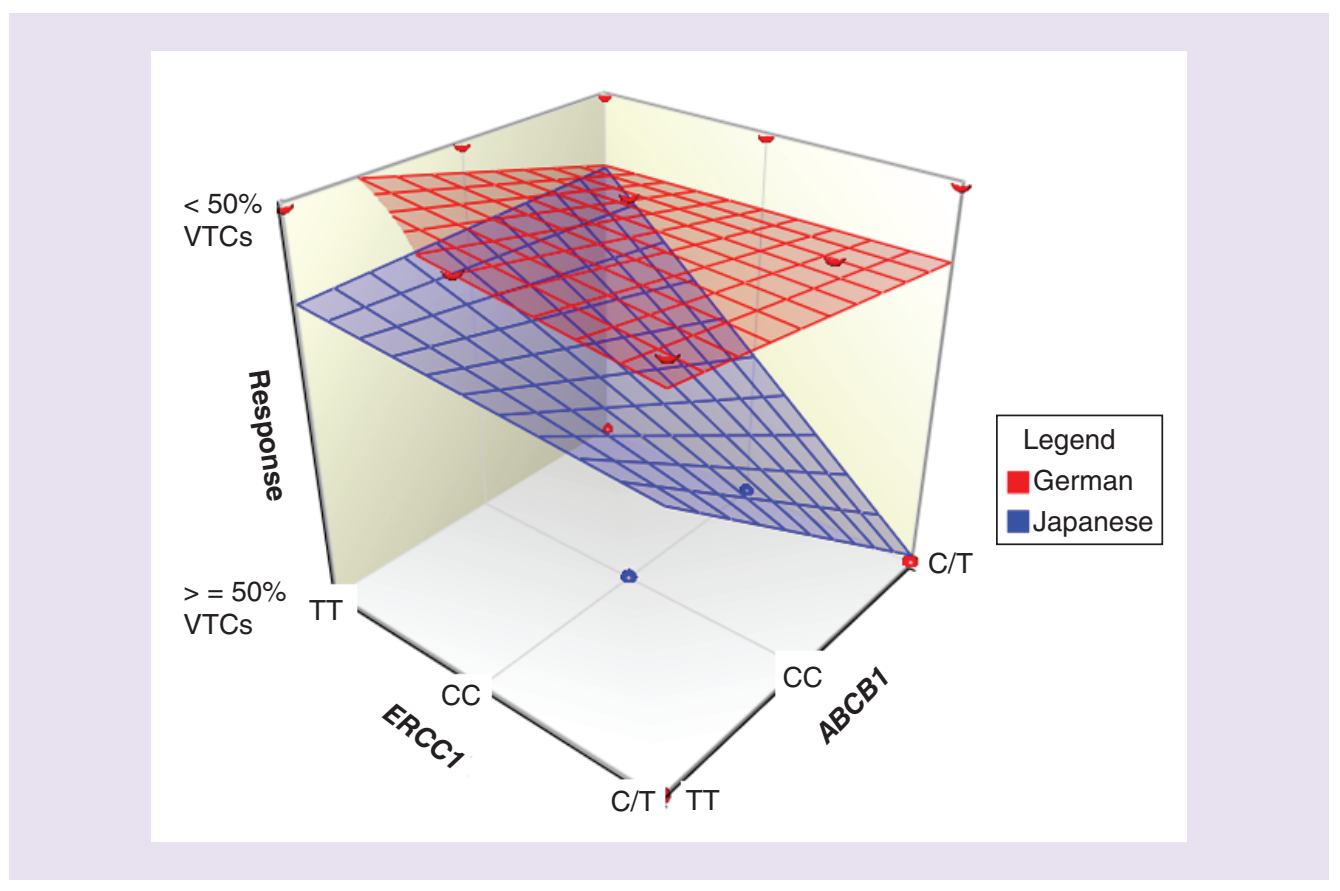

Figure 2. Graphical representation of multivariate analysis. Influence of ERCC1 rs16115 and $A B C B 1$ C3435T rs 1045642 to the response rate after neoadjuvant chemotherapy differ between Japanese and German patients with squamous cell carcinoma of the esophagus. Japan: response yes $=0.62+0.14 * E R C C 1+0.19 * A B C B 1-$

$0.18 E R C C 1 * A B C B 1$. Germany: Response yes $=1.25-0.99 * E R C C 1-0.15 * A B C B 1+0.03 E R C C 1 * A B C B 1$ with ERCC1 $\mathrm{rs} 16115 \mathrm{TT}=1, \mathrm{CC}=2$ and $\mathrm{C} / \mathrm{T}=3$ and $A B C B 1 \mathrm{C} 3435 \mathrm{~T} \mathrm{rs} 1045642 \mathrm{TT}=1, \mathrm{CC}=2$ and $\mathrm{C} / \mathrm{T}=3$.

VTC: Vital tumor cell.

greatest risk of non-response and TT ERCC1 combined with TT $A B C B 1$ the lowest risk for non-response.

The influence of the three SNPs on ypN0-category was significantly different for $E R C C 1 \mathrm{C} / \mathrm{T}(\mathrm{p}<0.002)$ and for GNAS C/T ( $\mathrm{p}<0.005)$ between Japanese and German patients.

Prognosis of Japanese \& German patients with advanced ESCC after neoadjuvant therapy

The prognosis was not significantly different between German and Japanese patients with advanced ESCC after preoperative therapy (Figure 3). Median survival rate for Japanese patients was 1.4 years (95\% CI: 0.9-3.9 years) and for German patients 2.0 years (95\% CI: $1.3-2.5$ years). 


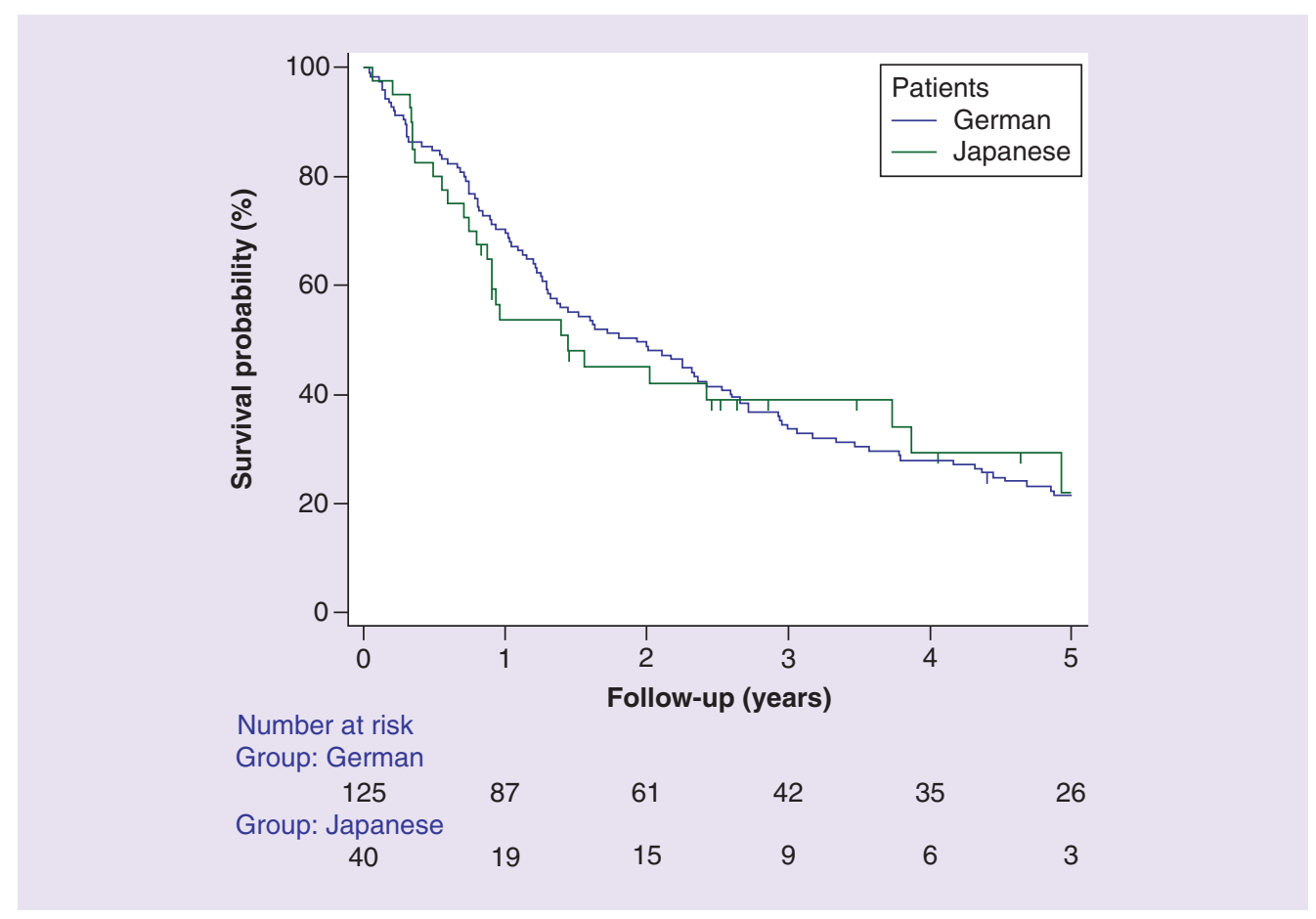

Figure 3. Kaplan-Meier survival curves comparing Japanese and German patients with advanced squamous cell carcinoma of the esophagus treated with neoadjuvant therapy based on cisplatin/5-FU followed by transthoracic esophagectomy.

\section{Discussion}

In this study, we compared especially patients with advanced esophageal squamous cell carcinoma from Japan and Germany. Both groups were treated with preoperative chemotherapy (5-FU and cisplatin) with or without radiation. We found that patients from Japan had significantly less often major response to this therapy compared with German patients. Response to neoadjuvant therapy was defined as reduction of the primary tumor from clinical T3-tumor to ypT0 or ypT1. Only 7 of 40 patients (18\%) from Japan showed ypT0-1, in contrast to 46 of 127 patients $(36 \%)$ from Germany ( $\mathrm{p}<0.04)$. In addition, more than half of the patients from Japan $(52 \%)$ were non-responder, defined as less than $50 \%$ reduction of the primary tumor or progression, compared with the patients from Germany with only $16 \%(\mathrm{p}<0.0001)$.

Further, we compared the regression of lymph node metastasis measured as the rate of patients with no lymph node metastasis after neoadjuvant therapy - ypN0. And again, Japanese patients showed significantly less often ypN0 (28\%) compared with German patients with 54\% ypN0.

The most appropriate explanation for the lower response rate in the Japanese patients is the lower frequency of patients with chemoradiation compared with German patients. Prospective randomized studies showed that chemoradiation is more effective than only chemotherapy in Caucasian patients with ESCC. Therefore, the German S3-guideline strongly recommends neoadjuvant chemoradiation and not chemotherapy only in ESCC [10,27]. According to the Guidelines for Diagnosis and Treatment of Carcinoma of the Esophagus in Japan, the standard treatment of esophageal cancer with clinical stage II/III is preoperative chemotherapy and radical resection. Currently, there is no firm basis for a recommendation of neoadjuvant chemoradiation [9]. Therefore, we included patients from Japan with chemotherapy with and without radiation. The rate of response was not significantly different after chemotherapy with or without radiation in Japanese patients.

There are several studies comparing the results of chemotherapy in patients with different tumor entities between Caucasian and Asian patients $[3,28,29]$. Chuah et al. studied the treatment with S-1 chemotherapy for patients with various tumor entities comparing Japanese and Caucasian patients [28]. S-1 is an oral fluoropyrimidine antineoplastic agent that is converted by CYP2A6 to 5-FU. They prospectively studied the pharmacokinetics and 
pharmacodynamics of S-1 and found no significant difference in 5-FU exposure between Caucasians and East Asians [28].

Consequently, we looked for further factors of influence to response. The reasons for the different response to neoadjuvant therapy may be multifactorial. Different factors have been described to influence drug response, including age, sex, ethnicity, comorbidity, drug interactions and especially genetics [30,31]. Several studies described the influence of polymorphism - like ERCC1 and $A B C B$ - to the response after chemotherapy or the prognosis of patients with esophageal carcinoma [5,13,15,16,22,23,32]. Therefore, we analyzed three SNPs, ERCC1 rs11615-SNP, $A B C B 1$ rs1045642 SNP and GNAS T393C rs7121 SNP from ESCC patients treated with comparable surgical and neoadjuvant therapy from two countries.

There are several reports about the frequency of these SNPs in the Japanese and European normal population $[14,33]$. These data show a significantly different distribution of the ERCC1 rs11615 genotypes between the Japanese and German normal populations ( $\mathrm{p}<0.0001$ ). Similar results were shown for $A B C B 1 \mathrm{C} 3435 \mathrm{~T}$ rs 1045642 $(\mathrm{p}<0.0001)$ in contrast to the distribution of the genotypes of GNAS SNP which was not different between the Japanese and German normal populations. The frequencies of all three SNPs analyzed in the patients were not different to published data from normal subjects in the corresponding populations (Table 2). Therefore, these SNPs are not risk factors for ESCC, but they may be relevant for response to chemotherapy.

Patients in both centers were treated with similar protocol of chemotherapy with 5-FU/cisplatin. Cisplatin is a platinum analog commonly used for many cancers. It binds to DNA, forms DNA adducts and inhibits DNA replication [17]. DNA repair mechanisms play an important role for response to cisplatin. Several studies have analyzed the influence of $E R C C 1$ or $A B C B 1$ SNPs to the response in patients treated with this chemotherapy $[13,33]$.

The meta-analysis from Ma et al. examined the association between the ERCC1 rs11615 polymorphism and the clinical outcomes of patients with different tumors that were treated by chemotherapy [33]. A stratified analysis indicated that the ERCC1 rs $11615 \mathrm{~T}$ allele was a biomarker of low therapeutic response in Asian gastric cancer patients. In addition, they found ethnic discrepancy in the prognosis between Asians and Caucasians and ethnic differences in the effect of the ERCC1 allele [34].

$E R C C 1$ is part of the nucleotide excision repair (NER) complex involved in repair of radiation and platinuminduced inter- and intrastrand crosslinks. Since efficient DNA repair capacity seems to be a critical mechanism of resistance to platinum drugs and radiation, we conclude that the NER pathway is important for therapy outcome [22]. The different frequency of ERCC1 rs11615 between both populations may explain partly the lower response rate in Japanese patients. In our study with ESCC patients only $12 \%$ of the Japanese patients with the CC genotype showed good response of the primary tumor (ypT0-1), but this rate was lower than in German patients with 35\%. About half of the Japanese population had the CC genotype of ERCC1 rs11615 in contrast to the German population with $13 \%$ CC genotype.

We show that the estimated frequencies of $A B C B 1$ C3453T genotypes in Japanese and Caucasian are different. These results are comparable to those of other studies [32]. The reasons are not really clear. $A B C B 1$ is understood to play an important role in the detoxification systems of normal tissues. Therefore, different environment factors are discussed [32].

In an earlier study, we observed that the CC genotype of $A B C B 1$ rs1045642 was associated with lymph node metastasis and distant metastasis in patients with SCC or AC of the esophagus [16]. These data were confirmed in the present study for ESCC patients from Japan with 80\% LNM and for German ESCC patients with 57\% lymph node metastasis (LNM). It is remarkable that half of the Japanese patients show the CC genotype $A B C B 1$ rs 1045642 in contrast to the German population with only $15 \%$. This may be one explanation for the significantly lower rate of ypN0 after neoadjuvant chemotherapy in Japanese ESCC patients compared with German ESCC patients. In addition, the CC genotype in the Japanese patients was significantly higher compared with the Japanese normal population. These different frequencies of the genotypes of $A B C B 1$ may be one causal relationship to the development of ESCC in Japanese patients.

Wu et al. studied relevant polymorphisms in the cisplatin pathway for Caucasian patients with ESCC [15]. One of these is the above-mentioned SNP rs1045642, a silent substitution in codon 3435 (C3435T) of the $A B C B 1$ gene. They found that the variant alleles $(\mathrm{CT}+\mathrm{TT})$ of $\mathrm{C} 3435 \mathrm{~T}$ were associated with reduced recurrence rate (hazard ratio $=0.25$ ) and improved survival (hazard ratio $=0.44)$ [15]. One explanation for these results is that the $\mathrm{T}$ allele seems responsible for slower metabolism of drugs like cisplatin and 5-FU [16]. Okuno et al. studied the same $A B C B 1$ SNP in 31 Japanese ESCC patients clinical stage T1-T4, N0 or N+, M0 treated with a 5-FU/CDDP-based CRT at Kobe University Hospital [5]. They found no correlation between genotypes of $A B C B 1$ rs 1045642 and response 
to chemoradiation or prognosis. One reason for this finding may be the high rate of cT1-2 tumors with $49 \%$. The rate of clinical complete response in this study was $56 \%$. This complete response was evaluated by endoscopy and the chest and abdominal CT scan 4 weeks after the last course before surgery. This kind of response evaluation is not fully accurate [35].

The strength of our study is the standardized high quality of response measurement by histopathologic evaluation of tumor in the resected specimen. There are several reports about the differences between clinical and histopathological response assessment [35-37]. Further, the indication to the therapy (clinical advanced primary tumor), the surgical procedure (transthoracic esophagectomy with two field lymphadenectomy) and the drugs for neoadjuvant therapy (5-FU/cisplatin) were comparable between the two hospitals.

Of course, there are some limitations:

- All data were analyzed for patients with esophagectomy. Therefore, patients with distant metastasis during the course of chemotherapy were not included. For German patients the rate of nonsurgical treatment after chemoradiation caused by progression or toxicity is about $13 \%$ [7]. The rate in Japanese patients may be higher;

- We have not analyzed how many patients in each group had reduced doses of chemotherapy. Several studies have shown that Caucasian patients had higher risk of toxicity than Asian patients [29,38]. Therefore, the response rate in Caucasian patients could be lower as a result of lower doses of chemotherapy, but this possible effect supports our findings;

- In this histopathological workup, we looked for the tumor regression in the primary tumor and the lymph nodes. But we have no data about the preoperative course of chemotherapy and its correlation with the SNPs;

- Another bias may the different regimen of chemotherapy between the two countries. Chemotherapy dose for Japanese is reduced to $70 \%$ of Germans as total dose. The chemoradiotherapy regimens in Japan were performed according to the Japan Esophageal Oncology Group [39]. Daily continuous infusion of cisplatin was not associated with higher response or lower toxicity than those seen with the high-dose bolus or multibolus treatment regimens by JECOG [40]. And the dose of daily fractions in radiation was different 2.0 and 1.8 Gy. But both of total dose were 40 Gy using a multiple field technique. We think that it was worth comparing between two groups.

\section{Conclusion \& future perspective}

The results of this study showed that Japanese and German patients with ESCC have different response rates to neoadjuvant chemotherapy with 5-FU/cisplatin. This result could be partly explained by different expression of ERCC1 and ABCB1 SNPs in the Japanese and German populations. The data are relevant for further pharmacotherapeutic research.

\section{Summary points}

- We hypothesized that there are ethnic differences in the molecular cancer biology and in the rate of response to radio/chemotherapy for squamous cell carcinoma of the esophagus (ESCC).

- Our study confirmed that the response after neoadjuvant chemotherapy (5-FU/cisplatin) with or without radiation was significantly lower in Japanese patients than in German patients (Table 3).

- We analyzed the therapeutic results comparing the data of patients with ESCC from Japan and Germany according to the frequency of three SNPs - ERCC1 rs11615, ABCB1 C3435T rs1045642 and G-protein rs7121 by real-time PCR by TaqMan 7900HT.

- Results showed that the distribution of the genotypes of ERCC1 rs16115 ( $p<0.0001)$ and of ABCB1 C3435T rs1045642 ( $p=0.002$ ) was significantly different between German and Japanese patients (Table 1).

- Comparing the published frequency of these three SNPs in Japanese and European normal population the CC and TT genotypes of ERCC1 and $A B C B 1$ was significantly different between both populations (Table 2).

- The frequency of the genotypes was not different between the patients and the normal population. Only patients from Japan showed higher frequency of CC $A B C B 1$ compared with Japanese normal population (Table 2).

- The influence of the genotypes of $A B C B 1$ and $E R C C 1$ on response varied between patients from Japan and Germany (Figure 2) and correlates with its different frequency in the patient's group.

- Lower response rate to neoadjuvant chemotherapy with 5-FU/cisplatin in Japanese compared with German patients with ESCC can be partly explained by different expressions of ERCC1 and ABCB1 SNPs in the patient groups. 


\section{Acknowledgments}

The authors would like to thank the study coordinators E Bollschweiler, U Warnecke-Eberz and AH Hoelscher for their support in this work.

\section{Financial \& competing interests disclosure}

This work was supported by the Department of General, Visceral and Cancer Surgery, Center for Integrated Oncology, University Hospital of Cologne. The authors have no other relevant affiliations or financial involvement with any organization or entity with a financial interest in or financial conflict with the subject matter or materials discussed in the manuscript apart from those disclosed.

No writing assistance was utilized in the production of this manuscript.

\section{Ethical conduct of research}

The authors state that they have obtained appropriate institutional review board approval or have followed the principles outlined in the Declaration of Helsinki for all human or animal experimental investigations. In addition, for investigations involving human subjects, informed consent has been obtained from the participants involved.

\section{Open access}

This work is licensed under the Attribution-NonCommercial-NoDerivatives 4.0 Unported License. To view a copy of this license, visit http://creativecommons.org/licenses/by-nc-nd/4.0/

\section{References}

Papers of special note have been highlighted as: $\bullet$ of interest; $\bullet \bullet$ of considerable interest

1. Abnet CC, Arnold M, Wei WQ. Epidemiology of esophageal squamous cell carcinoma. Gastroenterology 154(2), 360-373 (2018).

2. Bollschweiler E, Holscher AH. Carcinoma of the esophagus - actual epidemiology in Germany. Onkologie 24(2), 180-184 (2001).

3. Hashiguchi $\mathrm{Y}$, Hase $\mathrm{K}$, Ueno $\mathrm{H}$ et al. Impact of race/ethnicity on prognosis in patients who underwent surgery for colon cancer: analysis for white, African and East Asian Americans. Ann. Surg. Oncol. 19(5), 1517-1528 (2012).

4. Ikoma N, Blum M, Chiang YJ et al. Race is a risk for lymph node metastasis in patients with gastric cancer. Ann. Surg. Oncol. 24(4), 960-965 (2017).

5. Okuno T, Tamura T, Yamamori M et al. Favorable genetic polymorphisms predictive of clinical outcome of chemoradiotherapy for stage II/III esophageal squamous cell carcinoma in Japanese. Am. J. Clin. Oncol. 30(3), 252-257 (2007).

6. Kutup A, Nentwich MF, Bollschweiler E et al. What should be the gold standard for the surgical component in the treatment of locally advanced esophageal cancer: transthoracic versus transhiatal esophagectomy. Ann. Surg. 260(6), 1016-1022 (2014).

7. Holscher AH, Bollschweiler E, Bogoevski D et al. Prognostic impact of neoadjuvant chemoradiation in cT3 oesophageal cancer - a propensity score matched analysis. Eur. J. Cancer 50(17), 2950-2957 (2014).

8. van Hagen P, Hulshof MC, van Lanschot JJ et al. Preoperative chemoradiotherapy for esophageal or junctional cancer. N. Engl. J. Med. 366(22), 2074-2084 (2012).

9. Kuwano H, Nishimura Y, Oyama T et al. Guidelines for diagnosis and treatment of carcinoma of the esophagus April 2012 edited by the Japan Esophageal Society. Esophagus 12(1), 1-30 (2015).

10. Porschen R, Fischbach W, Gockel I et al. S3-Leitlinie - diagnostik und therapie der plattenepithelkarzinome und adenokarzinome des osophagus. Z Gastroenterol 57(3), 336-418 (2019).

11. Evans WE, Relling MV. Pharmacogenomics: translating functional genomics into rational therapeutics. Science 286(5439), 487-491 (1999).

12. Gusella M, Giacopuzzi S, Bertolaso L et al. Genetic prediction of long-term survival after neoadjuvant chemoradiation in locally advanced esophageal cancer. Pharmacogenomics J. 17(3), 252-257 (2017).

13. Vrana D, Hlavac V, Brynychova V et al. ABC transporters and their role in the neoadjuvant treatment of esophageal cancer. Int. J. Mol. Sci. 19(3), 868 (2018).

14. Findlay JM, Middleton MR, Tomlinson I. A systematic review and meta-analysis of somatic and germline DNA sequence biomarkers of esophageal cancer survival, therapy response and stage. Ann. Oncol. 26(4), 624-644 (2015).

15. Wu X, Gu J, Wu TT et al. Genetic variations in radiation and chemotherapy drug action pathways predict clinical outcomes in esophageal cancer. J. Clin. Oncol. 24(23), 3789-3798 (2006).

16. Narumiya K, Metzger R, Bollschweiler E et al. Impact of $A B C B 1 \mathrm{C} 3435 \mathrm{~T}$ polymorphism on lymph node regression in multimodality treatment of locally advanced esophageal cancer. Pharmacogenomics 12(2), 205-214 (2011).

17. Metzger R, Bollschweiler E, Holscher AH et al. ERCC1: impact in multimodality treatment of upper gastrointestinal cancer. Future Oncol. 6(11), 1735-1749 (2010). 
18. Miyazono F, Metzger R, Warnecke-Eberz U et al. Quantitative c-erbB-2 but not c-erbB-1 mRNA expression is a promising marker to predict minor histopathologic response to neoadjuvant radiochemotherapy in oesophageal cancer. Br. J. Cancer 91(4), 666-672 (2004).

19. Warnecke-Eberz $\mathrm{U}$, Vallbohmer $\mathrm{D}$, Alakus $\mathrm{H}$ et al. ERCC1 and XRCC1 gene polymorphisms predict response to neoadjuvant radiochemotherapy in esophageal cancer. J. Gastrointest. Surg. 13(8), 1411-1421 (2009).

20. Brabender J, Vallbohmer D, Grimminger P et al. ERCC1 RNA expression in peripheral blood predicts minor histopathological response to neoadjuvant radio-chemotherapy in patients with locally advanced cancer of the esophagus. J. Gastrointest. Surg. 12(11), 1815-1821 (2008).

21. Metzger R, Warnecke-Eberz U, Alakus $\mathrm{H}$ et al. Neoadjuvant radiochemotherapy in adenocarcinoma of the esophagus: ERCC1 gene polymorphisms for prediction of response and prognosis. J. Gastrointest. Surg. 16(1), 26-34; discussion 34 (2012).

22. Bollschweiler E, Holscher AH, Herbold T et al. Molecular markers for the prediction of minor response to neoadjuvant chemoradiation in esophageal cancer: results of the prospective Cologne Esophageal Response Prediction (CERP) study. Ann. Surg. 264(5), 839-846 (2016).

-• ERCC1-SNP in combination with mRNA ERCC1, DPYD and ERBB2 from pretherapeutic endoscopic biopsies can predict minor response to chemoradiation, as a basis for individualized therapy of advanced esophageal cancer.

23. Alakus H, Bollschweiler E, Holscher AH et al. Homozygous GNAS 393C-allele carriers with locally advanced esophageal cancer fail to benefit from platinum-based preoperative chemoradiotherapy. Ann. Surg. Oncol. 21(13), 4375-4382 (2014).

- Patients with a locally advanced esophageal cancer and an homozygous GNAS 393C genotype do not benefit from platinum-based neoadjuvant chemoradiotherapy, indicating that these patients should be treated by alternative treatment strategies.

24. Puetz K, Bollschweiler E, Semrau R et al. Neoadjuvant chemoradiation for patients with advanced oesophageal cancer - which response grading system best impacts prognostic discrimination? Histopathology 74(5), 731-743 (2019).

25. Abbasi $\mathrm{R}$, Ramroth $\mathrm{H}$, Becher $\mathrm{H}$ et al. Laryngeal cancer risk associated with smoking and alcohol consumption is modified by genetic polymorphisms in ERCC5, ERCC6 and RAD23B but not by polymorphisms in five other nucleotide excision repair genes. Int. J. Cancer 125(6), 1431-1439 (2009).

26. Hashiguchi M, Shimizu M, Hakamata J et al. Genetic polymorphisms of enzyme proteins and transporters related to methotrexate response and pharmacokinetics in a Japanese population. J. Pharm. Health Care Sci. 2, 35 (2016).

- The variations in allelic frequencies in different ethnic and/or population groups in healthy adults and rheumatoid arthritis (RA) patients may contribute to individual variations in methotrexate (MTX) response and toxicity.

27. Holscher AH, Gockel I, Porschen R. Updated German S3 guidelines on esophageal cancer and supplements from a surgical perspective. Chirurg 90(5), 398-402 (2019).

28. Chuah B, Goh BC, Lee SC et al. Comparison of the pharmacokinetics and pharmacodynamics of S-1 between Caucasian and East Asian patients. Cancer Sci. 102(2), 478-483 (2011).

29. Shirao K, Hoff PM, Ohtsu A et al. Comparison of the efficacy, toxicity and pharmacokinetics of a uracil/tegafur (UFT) plus oral leucovorin (LV) regimen between Japanese and American patients with advanced colorectal cancer: joint United States and Japan study of UFT/LV. J. Clin. Oncol. 22(17), 3466-3474 (2004).

30. Syn NL, Yong WP, Lee SC et al. Genetic factors affecting drug disposition in Asian cancer patients. Expert Opin. Drug Metab. Toxicol. 11(12), 1879-1892 (2015).

31. Bollschweiler E, Holscher AH, Metzger R. Histologic tumor type and the rate of complete response after neoadjuvant therapy for esophageal cancer. Future Oncol. 6(1), 25-35 (2010).

32. Komoto C, Nakamura T, Sakaeda T et al. MDR1 haplotype frequencies in Japanese and Caucasian and in Japanese patients with colorectal cancer and esophageal cancer. Drug Metab. Pharmacokinet. 21(2), 126-132 (2006).

- There is a considerable difference in the frequencies of haplotype including A2677 polymorphism between Japanese and Caucasion.

33. Ma SC, Zhao Y, Zhang T et al. Association between the ERCC1 rs11615 polymorphism and clinical outcomes of oxaliplatin-based chemotherapies in gastrointestinal cancer: a meta-analysis. Onco. Targets Ther. 8, 641-648 (2015).

34. Zazuli Z, Vijverberg S, Slob E et al. Genetic variations and cisplatin nephrotoxicity: a systematic review. Front. Pharmacol. 9,1111 (2018).

35. Schneider PM, Metzger R, Schaefer H et al. Response evaluation by endoscopy, rebiopsy and endoscopic ultrasound does not accurately predict histopathologic regression after neoadjuvant chemoradiation for esophageal cancer. Ann. Surg. 248(6), 902-908 (2008).

36. Uberoi GS, Uberoi AS, Bhutani MS. Endoscopic and imaging predictors of complete pathologic response after chemoradiation for esophageal cancer. Curr. Gastroenterol. Rep. 19(11), 57 (2017).

37. van Rossum PS, Goense L, Meziani J et al. Endoscopic biopsy and EUS for the detection of pathologic complete response after neoadjuvant chemoradiotherapy in esophageal cancer: a systematic review and meta-analysis. Gastrointest. Endosc. 83(5), 866-879 (2016).

38. Haller DG, Catalano PJ, MacDonald JS et al. Phase III study of fluorouracil, leucovorin and levamisole in high-risk stage II and III colon cancer: final report of Intergroup 0089. J. Clin. Oncol. 23(34), 8671-8678 (2005). 
39. Ishida K, Ando N, Yamamoto S et al. Phase II study of cisplatin and 5-fluorouracil with concurrent radiotherapy in advanced squamous cell carcinoma of the esophagus: a Japan Esophageal Oncology Group (JEOG)/Japan Clinical Oncology Group trial (JCOG9516). Jpn. J. Clin. Oncol. 34(10), 615-619 (2004).

40. Hayashi $\mathrm{K}$, Ando N, Watanabe $\mathrm{H}$ et al. Phase II evaluation of protracted infusion of cisplatin and 5-fluorouracil in advanced squamous cell carcinoma of the esophagus: a Japan Esophageal Oncology Group (JEOG) Trial (JCOG9407). Jpn. J. Clin. Oncol. 31(9), 419-423 (2001). 